\title{
Vasospasm following low-velocity penetrating pediatric intracranial trauma
}

\author{
Alysa Almojuela ${ }^{1 *} \mathbb{D}$, Zul Kaderali², James McEachern ${ }^{3}$, Colin Kazina ${ }^{1}$ and Demitre Serletis ${ }^{4,5}$
}

\begin{abstract}
Background: BB guns or non-powder guns created in the modern era are able to reach exceedingly fast velocities as a result of advances in compressed-gas technology. While missile penetrating trauma has been well documented in neurosurgical literature, penetrating intracranial injury secondary to non-powder guns, along with their associated complications and treatments, is not well described, and even less so in the pediatric population.

Case presentation: Here, we describe an unusual case of a 6-year-old Indigenous child who was accidentally shot with a BB gun to the head. He subsequently developed delayed acute right-sided weakness due to symptomatic vasospasm. His symptoms resolved following hypertensive therapy, balloon angioplasty intervention, and intra-arterial milrinone therapy.

Conclusions: This case highlights the unique complication of delayed symptomatic vasospasm in a child following a non-missile intracranial penetrating injury. Intracranial vasospasm needs to be considered in the presence of delayed neurological deficit given its potential reversibility. This case also emphasizes the importance of gun safety and education when handling and storing these potential weapons around children.
\end{abstract}

Keywords: BB gun, Pediatric, Penetrating brain injury, Cerebral vasospasm, Case report

\section{Background}

Penetrating intracranial injuries pose unique challenges to medical and neurosurgical management, particularly in the pediatric patient cohort and in low-velocity type injuries, such as those inflicted by BB guns (air guns used to fire metallic ball projectiles or pellets). Between 2003 and 2013 in the USA, 200,645 nonfatal pellet gun injuries were reported, with nearly two-thirds $(127,742)$ occurring in children [1]. Further, hospitalizations due to injuries inflicted by non-powder guns cost more than 10 million US dollars annually; of hospitalized patients, half require a major surgical procedure [2]. Despite the potential morbidity associated with non-powder guns, approximately 3.2 million $\mathrm{BB}$ and/or pellet guns continue

\footnotetext{
*Correspondence: umalmoja@myumanitoba.ca

${ }^{1}$ Section of Neurosurgery, Department of Surgery, University of Manitoba, Winnipeg, MB, Canada

Full list of author information is available at the end of the article
}

to be sold in the USA every year [3], and while variable local laws exist in some states, there are no federal laws to regulate their sales or use [2].

Whereas high-velocity injuries are often fatal, lowvelocity injuries such as those caused by non-powder guns are less likely to be lethal and are associated with complications such as infection, pseudoaneurysm formation, and, rarely, symptomatic vasospasm. This cohort of patients therefore requires special consideration for follow-up and management by health professionals, including neurosurgeons. Here, we present an unusual case of a child who sustained a low-velocity penetrating intracranial injury from a BB gun, which caused symptomatic intracranial vasospasm, and was successfully treated with hypertensive therapy, balloon angioplasty, and intra-arterial milrinone therapy. original author(s) and the source, provide a link to the Creative Commons licence, and indicate if changes were made. The images or other third party material in this article are included in the article's Creative Commons licence, unless indicated otherwise in a credit line to the material. If material is not included in the article's Creative Commons licence and your intended use is not permitted by statutory regulation or exceeds the permitted use, you will need to obtain permission directly from the copyright holder. To view a copy of this licence, visit http://creativecommons.org/licenses/by/4.0/. The Creative Commons Public Domain Dedication waiver (http://creativeco mmons.org/publicdomain/zero/1.0/) applies to the data made available in this article, unless otherwise stated in a credit line to the data. 


\section{Methods}

Formal approval to publish this case report was obtained from the authors' institutional research ethics board. Consent was also obtained from the patient's mother to publish the study as a case report. The intent of the study and possible risks of publication related to patient confidentiality were discussed.

A literature review was conducted by searching PubMed for studies related to pediatric low-velocity penetrating intracranial injury, BB guns, pellet guns, and intracranial vasospasm. The retrieved studies, which included reviews, case series/reports, and retrospective studies, were reviewed by the primary author.

\section{Case presentation}

\section{Patient presentation}

A 6-year-old, previously healthy Indigenous male was accidentally shot in the left supraorbital area with a BB gun while playing at home. He was emergently flown to a tertiary trauma center for further management. On examination, his Glasgow Coma Scale (GCS) was 13 (E3V4M6). He displayed no focal neurological deficits. A small entry wound was visible just superior to his right eye, with no exit wound identified. Urgent computed tomography (CT) imaging (including CT angiography, or CTA) revealed two intracranial foreign bodies (that is, shrapnel), with
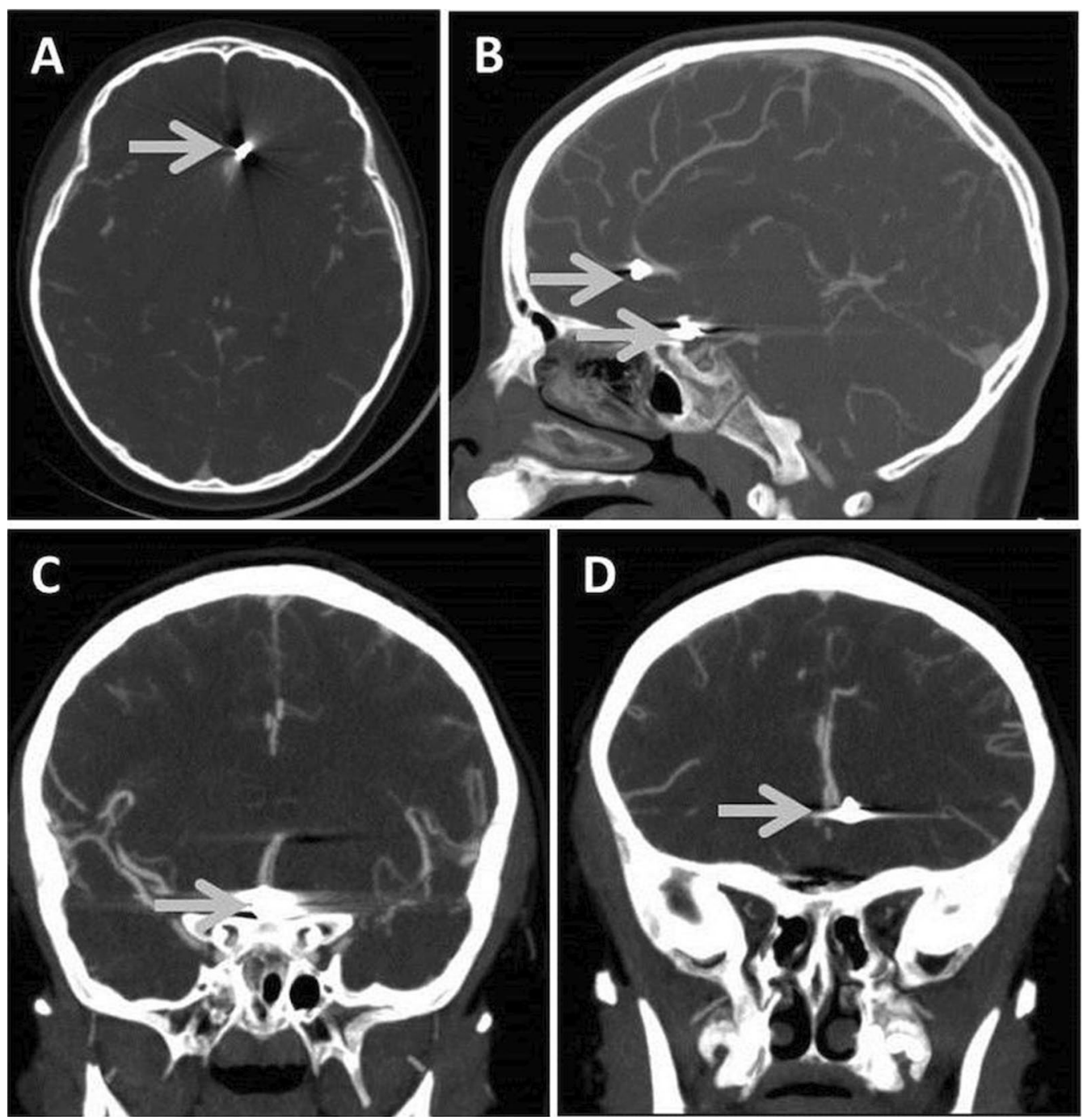

Fig. 1 Axial (A), sagittal (B), and coronal (C, D) views of CTA study, confirming the location of two intracranial, metallic shrapnel fragments from a BB pellet gun injury (shrapnel demarcated by gray arrows) 
surrounding subarachnoid hemorrhage in the Sylvian fissures, suprasellar cistern, ambient cisterns, and frontal lobes (Fig. 1A-D). Ventricular caliber was normal, and there was no evidence of parenchymal injury or ischemic stroke. CTA of the circle of Willis was limited due to metallic streak artifacts, but confirmed proximity of one piece of shrapnel $2 \mathrm{~mm}$ lateral to the left A2 segment in the frontal lobe. The second foreign body localized to the region of the anterior communicating artery. The distal vasculature appeared intact, with no signs of vasospasm. An early cerebral angiogram was performed, confirming these findings.

Given his neurological and hemodynamic stability, the patient was admitted for close clinical observation. $\mathrm{He}$ was started on phenytoin for seizure prophylaxis and antibiotic therapy (that is, empiric ceftriaxone, vancomycin, and metronidazole), given the nature of the penetrating traumatic injury. Seven days following admission to hospital, the patient acutely developed right-sided weakness affecting his arm (motor grade $4 / 5$ ) and leg (motor grade $4+/ 5$ ). He was otherwise hemodynamically stable, with minimal headache and normal level of consciousness. Urgent CTA imaging revealed narrowing of the left middle and anterior cerebral arteries, along with the supraclinoid portion of the left internal cerebral artery. The patient was thus transferred to the intensive care unit to initiate hypertensive therapy for vasospasm. Maintenance intravenous fluids were started, using fluid boluses and norepinephrine to maintain a mean arterial pressure (MAP) of greater than $90 \mathrm{mmHg}$, which improved his weakness.

The following day, though his weakness was improved, the patient became disoriented and developed expressive speech dysarthria. A cerebral angiogram was performed showing persistent vasospasm in the affected vessels, and balloon angioplasty of the left internal carotid, left middle cerebral, left anterior cerebral, and right anterior cerebral arteries was subsequently performed, as shown in Fig. 2A, B. Five milligrams of intra-arterial milrinone were delivered, and the patient was started on nimodipine at a dose of $15 \mathrm{mg}$ every 6 hours (roughly $1.5 \mathrm{mg}$ per $\mathrm{kg}$ per day). His MAP was maintained at greater than $90 \mathrm{mmHg}$ for 3 days, then greater than $85 \mathrm{mmHg}$ for 4 days, totaling 7 days of MAP-directed therapy. The patient's symptoms resolved, and repeat CTA imaging revealed radiographic resolution of the previously identified vasospasm in the affected vessels. The patient remained stable and was discharged from hospital 6 weeks post-admission.

\section{Discussion and conclusions}

Many of today's modern compressed-gas weapons generate enough muzzle velocity to easily penetrate the adult skull. While a common pellet weighing approximately $0.5 \mathrm{~g}$ requires a minimum velocity of only $825 \mathrm{ft} /$ second to penetrate the adult skull, some commercially

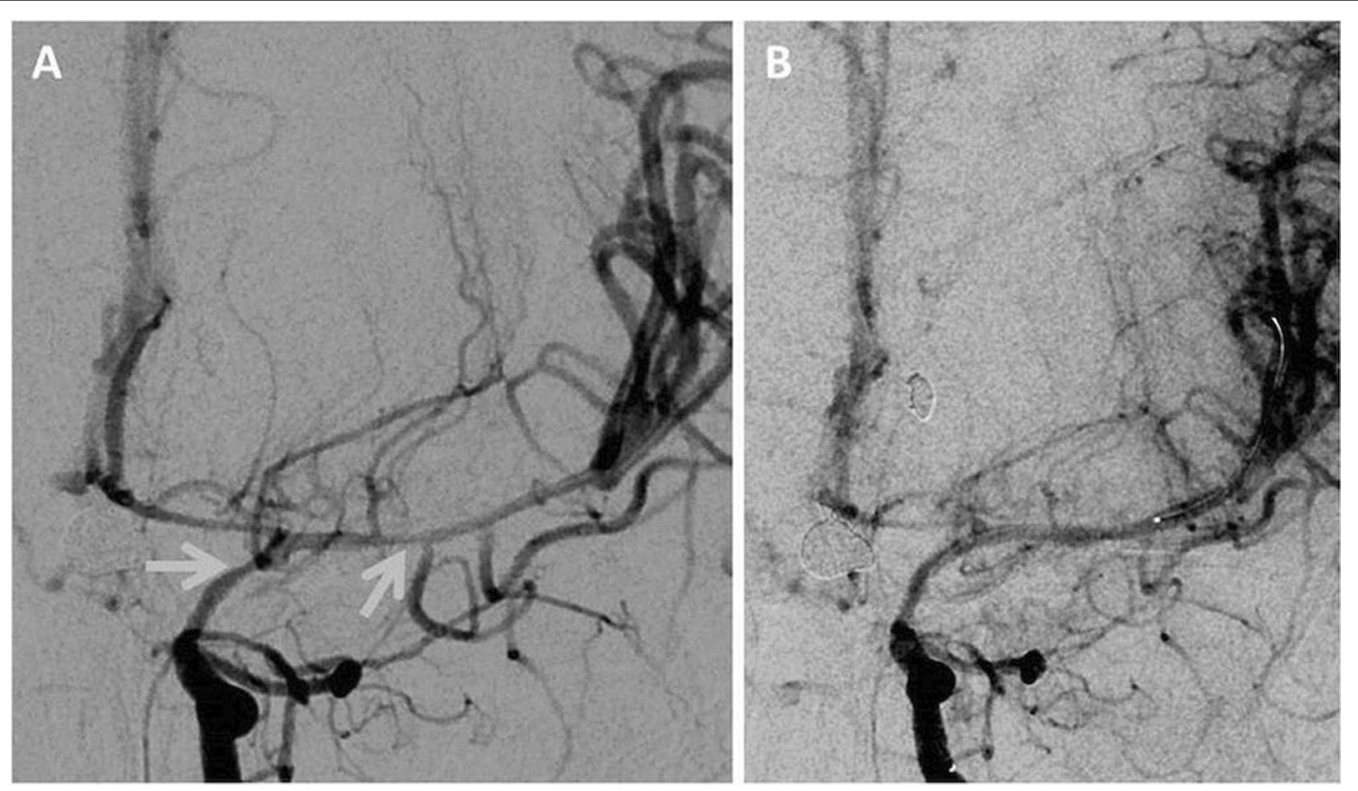

Fig. 2 A Anteroposterior view of a cerebral angiogram study showing radiographic evidence for vasospasm, with narrowing identified at the supraclinoid portion of the left internal cerebral artery and along the left middle cerebral artery (shown by gray arrows). B Radiographic improvement following injection of intra-arterial milrinone and balloon angioplasty 
available non-powder guns have muzzle velocities upwards of $1250 \mathrm{ft} / \mathrm{second}$ [3]. Moreover, there is a greater propensity for children to sustain severe injuries from these weapons secondary to their thinner skulls and soft tissues [3]. This case highlights the unique vascular complication of vasospasm following an unusual lowvelocity penetrating intracranial injury in a child.

In general, the most common vascular complication reported in low-velocity penetrating intracranial injuries is traumatic pseudoaneurysm formation, typically developing within the first 2-3 weeks of injury, but also known to occur in a delayed fashion [3-5]. Whereas pseudoaneurysm formation is a well-known sequela to this type of injury [4], vasospasm has been less studied in this context, and even less so in the pediatric population. Studies suggest that the onset of vasospasm typically begins between 2 and 5 days after injury, as in aneurysmal subarachnoid hemorrhage [6-8]. In the setting of traumatic subarachnoid hemorrhage, the underlying mechanism for the development of vasospasm largely relies on inflammatory pathways. As blood products are broken down by phagocytosis, free radical oxygen species are generated. These species then scavenge electron donors from the lipid bilayers of vascular endothelial and smooth muscle cell membranes, leading to dysfunction of vascular walls and upregulation of vasoconstrictors such as prostaglandins, serotonins, and thromboxin [7, 9]. These physiological changes collectively result in a net vasoconstrictive effect (that is, vasospasm) in the affected vessels, within the vicinity (and beyond) of the subarachnoid hemorrhage. The development of vasospasm in the case presented here may have been attributable to a combination of subarachnoid hemorrhage, direct vascular irritation or damage, and local inflammation due to the retained pellet fragments.

Importantly, the incidence and prevalence of vasospasm following traumatic brain injury has not been thoroughly studied, with only small cohorts reported in the literature. Kordestani et al. identified a $42.4 \%$ incidence of vasospasm using transcranial Doppler following cranial gunshot wounds in patients aged 15-50 years [6], and showed a trend towards favorable neurological outcome in patients without vasospasm. A favorable Glasgow Outcome Score (that is, good recovery or moderate disability) was observed in $47.4 \%$ of patients without evidence of vasospasm at least 3 months following injury, compared with a lower incidence (35.7\%) in those with vasospasm. Although not statistically significant $(p=0.12)$, this suggests that post-traumatic vasospasm may play a substantial role in the worsened outcomes reported for cases of low-velocity penetrating intracranial injury [6].

Within the pediatric literature, a prospective study of 69 children with traumatic brain injury [7] showed a prevalence of middle cerebral artery vasospasm (as diagnosed via Doppler ultrasound) of $8.5 \%$ in moderate traumatic brain injury patients (defined by GCS 9-12), and $33.5 \%$ in patients with severe traumatic brain injury $(\mathrm{GCS} \leq 8)$. Similarly, the prevalence of basilar artery vasospasm in moderate brain injury patients was $3 \%$, and much higher $(21 \%)$ in severe brain injury patients. Again, there was a trend towards improved neurological outcome in patients without vasospasm. In moderate traumatic brain injury, good neurological outcome (as defined by a pediatric-based Glasgow Outcome ScoreExtended $\geq 4$ at 1 month post-injury) was seen in $76 \%$ in patients without evidence for vasospasm, as compared with $40 \%$ in those with diagnosed vasospasm. In severe traumatic brain injury patients, good outcomes were observed in $29 \%$ of patients without vasospasm, as compared with only $15 \%$ of patients with diagnosed vasospasm [7].

Despite a relatively high prevalence of vasospasm diagnosed via diagnostic imaging, symptomatic vasospasm is thought to be rare. A retrospective study of 37 children with subarachnoid hemorrhage [10] demonstrated the prevalence of vasospasm to be $46 \%$ (as determined by angiography); despite this, only three children (8\%) manifested with clinically symptomatic vasospasm, all of whom had poor collateral vessels on vascular imaging. Thus, excellent collateralization of cerebral vasculature may be protective against vasospasm in children. Other factors that may explain some resilience in the pediatric cohort to develop clinically significant vasospasm may relate to clinical hemodynamic fluctuations, as well as the resilience of molecular pathways regulating the synthesis of vasoconstrictors and vasodilators such as nitric oxide [10].

Treatment of traumatic vasospasm is complex, as the standard treatment of hypertension, hypervolemia, and hemodilution often used in aneurysmal vasospasm can be detrimental in trauma patients with coexistent cerebral edema, and should be pursued cautiously [11]. Nimodipine, a dihydropyridine calcium channel blocker, is a standard part of therapy following aneurysmal subarachnoid hemorrhage in adults to prevent morbidity associated with delayed cerebral ischemia [12]. In children, robust studies evaluating the efficacy of nimodipine are lacking; however, it may be considered in the management of vasospasm [13]. Angioplasty and intra-arterial vasodilators have also been well described as effective treatment options in aneurysmal causes of vasospasm [14] but have not been thoroughly evaluated with respect to post-traumatic etiologies. These treatment modalities were extrapolated from the adult aneurysmal vasospasm literature and employed successfully in our case; however, their routine use in post-traumatic pediatric cases has yet 
to be rigorously studied. The utility of removing any foreign bodies to prevent vasospasm has also not been thoroughly studied and was avoided in this case owing to the patient's hemodynamic and neurologic stability.

Aside from vasospasm, important nonvascular complications following low-velocity penetrating intracranial injuries include infection $[5,15]$, with theoretically higher rates in low-velocity injuries where the penetrating pellet is considered unsterile. Moreover, post-traumatic seizures, known to arise in $30-50 \%$ of patients [15], are also common. Additional long-term consequences to consider include intracranial migration of $\mathrm{BB}$ pellets into other critical neurovascular structures, and lifelong contraindication to magnetic resonance (MR) imaging [3].

Strengths of this case report include the uniqueness of the case and the use of successful vasospasm treatment that resolved the patient's symptoms. This case report also has educational value in that it is hypothesis generating for the ideal treatment of traumatic intracranial vasospasm in pediatrics. Limitations include the single patient case and, thus, lack of generalizability. Given our single case, further studies are required to demonstrate the effectiveness of treatment (Additional file 1).

Overall, low-velocity penetrating intracranial injuries in the pediatric population are a source of significant morbidity. Symptomatic vasospasm in this context has not been well described, and its optimal treatment is unclear. Here, we describe a rare pediatric case exemplifying the unique complication of symptomatic vasospasm in the context of a penetrating injury by a low-velocity BB gun. Based on the findings presented here, vasospasm should be considered in the presence of a delayed onset of neurological deficits, and early management should be initiated given its potential reversibility.

\section{Abbreviations}

MR: Magnetic resonance; GCS: Glasgow Coma Scale; CT: Computed tomography; MAP: Mean arterial pressure; $\mathrm{mmHg}$ : Millimeters of mercury; $\mathrm{ft} / \mathrm{sec}$ : Feet per second.

\section{Supplementary Information}

The online version contains supplementary material available at https://doi. org/10.1186/s13256-022-03254-5.

Additional file 1. An additional file contains the completed CARE Checklist.

\section{Acknowledgements}

Not applicable.

\section{Authors' contributions}

AA was responsible for the acquisition and interpretation of data and drafting of the manuscript. ZK, JM, CK, and DS were responsible for analysis and interpretation of data. All authors read and approved the final manuscript.

\section{Funding}

This research did not receive any grants from funding agencies in the public, commercial, or not-for-profit sectors.

Availability of data and materials

Not applicable.

\section{Declarations}

\section{Ethics approval and consent to participate}

Ethics approval for the completion of this case report was granted by the Bannatyne Campus Research Ethics Board, University of Manitoba, Winnipeg.

\section{Consent for publication}

Written informed consent was obtained from the patient's legal guardian for publication of this case report and any accompanying images. A copy of the written consent is available for review by the Editor-in-Chief of this journal.

\section{Competing interests}

The authors declare that they have no competing interests.

\section{Author details}

'Section of Neurosurgery, Department of Surgery, University of Manitoba, Winnipeg, MB, Canada. '2 Division of Neurosurgery, Royal Columbian Hospital, New Westminster, BC, Canada. ${ }^{3}$ Department of Radiology, University of Manitoba, Winnipeg, MB, Canada. ${ }^{4}$ Department of Neurosurgery, Cleveland Clinic Foundation, Cleveland, OH, USA. ${ }^{5}$ Charles Shor Epilepsy Center, Neurological Institute, Cleveland Clinic, Cleveland, OH, USA.

Received: 29 October 2020 Accepted: 3 January 2022

Published online: 06 February 2022

\section{References}

1. Veenstra M, Prasad J, Schaewe H, Donoghue L, Langenburg S. Nonpowder firearms cause significant pediatric injuries. J Trauma Acute Care Surg. 2015;78(6):1138-42.

2. McLoughlin RJ, Stetson A, Green J, Hirsh MP, Cleary MA, Aidlen JT. Toy guns, real danger: an update on pediatric injury patterns related to nonpowder weapons. J Pediatr Surg. 2020;55(1):146-52.

3. Kumar R, Kumar R, Mallory GW, Jacob JT, Daniels DJ, Wetjen NM, et al. Penetrating head injuries in children due to BB and pellet guns: a poorly recognized public health risk. J Neurosurg Pediatr. 2016;17(2):215-21.

4. Alvis-Miranda HR, Rubiano AM, Agrawal A, Rojas A, Moscote-Salazar LR, Satyarthee GD, Calderon-Miranda WG, Hernandez NE, Zabaleta-Churio N. Craniocerebral gunshot injuries - a review of the current literature 2016. Bull Emerg Trauma. 2016;4(2):65-74.

5. Sweeney JM, Lebovitz JJ, Eller JL, Coppens JR, Bucholz RD, Abdulrauf SI. Management of nonmissile penetrating brain injuries: a description of three cases and review of the literature. Skull Base Rep. 2011;1 (1):39-46.

6. Kordestani RK, Counelis GJ, McBride DQ, Martin NA. Cerebral arterial spasm after penetrating craniocerebral gunshot wounds: transcranial Doppler and cerebral blood flow findings. Neurosurgery. 1997:41(2):351-9.

7. O'Brien NF, Maa T, Yeates KO. The epidemiology of vasospasm in children with moderate-to-severe traumatic brain injury. Crit Care Med. 2015;43(3):674-85.

8. O'Brien NF, Reuter-Rice KE, Khanna S, Peterson BM, Quinto KB. Vasospasm in children with traumatic brain injury. Intensive Care Med. 2010:36(4):680-7.

9. Macdonald RL, Weir BK. A review of hemoglobin and the pathogenesis of cerebral vasospasm. Stroke. 1991;22:971-82.

10. Moftakhar P, Cooke DL, Fullerton HJ, Ko NU, Amans MR, Narvid JA, et al. Extent of collateralization predicting symptomatic cerebral vasospasm among pediatric patients: correlations among angiography, transcranial Doppler ultrasonography, and clinical findings. J Neurosurg Pediatr. 2015;15(3):282-90.

11. Chamoun RB, Jea A. Traumatic intracranial and extracranial vascular injuries in children. Neurosurg Clin N Am. 2010;21(3):529-42. 
12. Seiler RW, Grolimund P, Zurbruegg HR. Evaluation of the calciumantagonist nimodipine for the prevention of vasospasm after aneurysmal subarachnoid hemorrhage a prospective transcranial Doppler ultrasound study. Acta Neurochir. 1987;85:7-16.

13. Heffren J, Mclntosh AM, Reiter PD. Nimodipine for the prevention of cerebral vasospasm after subarachnoid hemorrhage in 12 children. Pediatr Neurol. 2015;52(3):356-60

14. Pierot L, Aggour M, Moret J. Vasospasm after aneurysmal subarachnoid hemorrhage: recent advances in endovascular management. Curr Opin Crit Care. 2010;16(2):110-6.

15. Drosos E, Giakoumettis D, Blionas A, Mitsios A, Sfakianos G, Themistocleous M. Pediatric nonmissile penetrating head injury: case series and literature review. World Neurosurg. 2018;110:193-205.

\section{Publisher's Note}

Springer Nature remains neutral with regard to jurisdictional claims in published maps and institutional affiliations.

Ready to submit your research? Choose BMC and benefit from:

- fast, convenient online submission

- thorough peer review by experienced researchers in your field

- rapid publication on acceptance

- support for research data, including large and complex data types

- gold Open Access which fosters wider collaboration and increased citations

- maximum visibility for your research: over 100M website views per year

At BMC, research is always in progress.

Learn more biomedcentral.com/submissions 